\title{
Location-specific attentional control is also possible in the Simon task
}

\author{
Ronald Hübner $^{1} \cdot$ Shreyasi Mishra $^{1}$
}

Published online: 13 May 2016

(C) Psychonomic Society, Inc. 2016

\begin{abstract}
Goal-directed behavior usually requires mental control that directs attention to task-relevant information and ignores irrelevant information. For investigating how flexible this mechanism is, researchers have varied the proportion of congruent trials depending on some context, such as stimulus location. The corresponding studies revealed that attentional control indeed adapts to location-specific demands. However, until now, this flexibility has only been demonstrated for the Eriksen flanker task and for the Stroop task but not for the Simon task. Therefore, a Simon-task experiment was conducted in the present study, where the proportion of congruent trials differed for stimuli appearing above or below fixation, respectively. As a result, a reliable interaction between congruency and stimulus location was found. This demonstrates, for the first time, that location-specific control also is possible in the Simon task.
\end{abstract}

Keywords Simon task · Proportion congruent .

Context-specific control

\section{Introduction}

Cognitive control is a crucial prerequisite for goal-directed behavior. Accordingly, several conflict paradigms have been invented, such as the Stroop task (Steinhauser \& Hübner, 2009; Stroop, 1935), the Flanker task (Eriksen \& Schultz,

Ronald Hübner

ronald.huebner@uni-konstanz.de

Fachbereich Psychologie, Universität Konstanz, Fach D29, D-78457 Konstanz, Germany
1979; Hübner, Steinhauser, \& Lehle, 2010), and the Simon task (Lu \& Proctor, 1995; Simon, 1969; Töbel, Hübner, \& Stürmer, 2014), for investigating its mechanisms. In all these paradigms, task-relevant information is presented along with information that is task-irrelevant but nonetheless associated with a response. Trials for which the irrelevant information activates the correct or wrong response are referred to as "congruent" or "incongruent," respectively. A common observation is that responses are faster and more accurate on congruent trials than on incongruent ones. This congruency effect has been interpreted in the sense that certain stimuli are processed automatically.

An important question in this context is to what extent the processing of irrelevant information can be controlled and how the degree of control is determined. A widely applied method to investigate these issues is to vary the proportion of congruent trials relative to incongruent ones (Hasegawa \& Takahashi, 2013; Hommel, 1994; Logan \& Zbrodoff, 1979; Marble \& Proctor, 2000; Stürmer, Leuthold, Soetens, Schröter, \& Sommer, 2002; Toth et al., 1995). If proportion congruent (PC) is relatively high in a block of trials, then the congruency effect is usually increased compared with balanced proportions, whereas the effect is smaller when PC is relatively low. This listwide $\mathrm{PC}$ effect suggests that the degree of irrelevant-information processing is generally adjusted according to the overall demands in a block of trials. However, a great difficulty with this interpretation is that unbalanced proportions also produce unbalanced transition frequencies between congruent and incongruent trials. Because it is known that the congruency effect decreases or increases after an incongruent or congruent trial, respectively (Botvinick, Braver, Barch, Carter, \& Cohen, 2001; Gratton, Coles, \& Donchin, 1992), the list-wide PC effect can be explained, at least partly, by cumulative sequential effects. That is, a decreased list-wide congruency effect in blocks with a low proportion of congruent trials could simply result from the sequentially reduced congruency effect on the majority of trials. 
An analogue reasoning holds for the increased congruency effect in high-PC blocks. In any case, list-wide PC effects show that some control takes place, although it is unclear to what extent it proceeds by retroactive local adjustments or by a listwide attentional strategy.

In another line of research on mental control, the confounding of PC effects with sequential effects has been prevented by coupling PC with a specific context, such as stimulus location, or with specific stimulus attributes, such as color (Abrahamse, Duthoo, Notebaert, \& Risko, 2013; Crump, Gong, \& Milliken, 2006; Lehle \& Hübner, 2008). For instance, in a flanker-task study by Lehle and Hübner (2008), high and low PC were linked to specific stimulus colors, whereas overall PC was balanced in each block of trials. As a result, the congruency effect varied with stimulus color according to the corresponding PC. This item-specific PC effect demonstrates that information processing can be controlled proactively. Obviously, participants used item color as cue to predict whether the stimulus was likely to be congruent or incongruent and to immediately adjust the control parameters accordingly. However, Lehle and Hübner (2008) also found that this item/color-specific control was only possible when participants had learned the association between color and proportion before under blocked conditions (see also Vietze \& Wendt, 2009).

Such a preliminary learning is not necessary, if PC is coupled to stimulus location. Wendt, Kluwe, and Vietze (2008), for instance, presented the stimuli in a flanker task at one of four locations on a screen, each of which was related to a certain PC, whereas overall proportion was balanced. With this arrangement they found a context-specific PC effect, i.e., a variation of the congruency effect according to the PC linked to the individual locations. Crump et al. (2006) observed a similar locationspecific effect for the Stroop task. Interestingly, they also used stimulus shape as cue for PC, which could not be utilized for attentional control. Thus, location seems to play a special role for signifying the control demand.

Unfortunately, for some reason, location-specific PC effects have only been shown for the flanker task and the Stroop task but not yet for the Simon task. Perhaps it has been thought that the Simon task is inappropriate in this respect, because stimulus location already serves for irrelevant response activation. In this task participants have to indicate the feature (e.g., "red" or "blue") of a stimulus presented either to the left or right of fixation by pressing a "left" or "right" button. Although stimulus location is irrelevant, responses are usually faster and more reliable when the stimulus position is response compatible (congruent), i.e., ipsilateral to the required response, compared with when it is response incompatible (incongruent), i.e., contralateral to the correct response. Obviously, stimulus location automatically activates the corresponding response code and this activation is faster or initially stronger than that produced by the relevant stimulus attribute (Lu \& Proctor, 1995). In any case, this Simon effect demonstrates that task-irrelevant spatial information substantially can influence response selection.

Given the specific spatial layout of stimulus and response locations in the standard Simon task, and the corresponding congruency effect, it obviously makes little sense additionally to relate $\mathrm{PC}$ to the same locations. However, different PC conditions can spatially be separated along the vertical dimension. Such a method has already been applied by Proctor, Vu, and Marble (2003) to investigate spatialcompatibility effects. In one condition in their Experiment 3, they mixed a location-relevant task with a location-irrelevant (Simon) task. For the location-relevant task, white stimuli appeared left or right in a given row on the display. Depending on the compatibility condition, the participants had to respond to the left-right locations with a spatially compatible or incompatible key press, respectively. The location-irrelevant task was a usual Simon task, for which colored stimuli were presented in a separate row. Thus, stimulus location was $100 \%$ congruent or $100 \%$ incongruent in one row (location-relevant task) and $50 \%$ congruent in the other row (Simon task).

With this task-mixing method Proctor et al. (2003) found for the location-relevant task that the spatial-compatibility effect, i.e., the advantage of the compatible location-response mapping relative to the incompatible one, which is usually relatively large, was absent. Moreover, the Simon effect was increased when the location-response mapping for the location-relevant task was compatible, but reversed when it was incompatible. These results indicate that there was strong interference between the two tasks, despite the fact that the stimuli differed between the individual tasks in color and vertical location. This suggests that the participants had great difficulties to accomplish color- or location-specific control. Even more, in a further condition, where the stimuli of both tasks could randomly appear in any row, Proctor et al. (2003) found the same results. Thus, task interference was independent of whether the stimuli for the different tasks appeared in separate rows or not. This demonstrates that location-specific control was completely absent.

The results of Proctor et al. (2003) do not support the hypothesis that location-specific PC effects can be found in the Simon task. However, one must take into account that these researchers applied a procedure that differed in several respects from the paradigm usually applied in studies on location-specific PC effects. For instance, the participants had to switch between two tasks (color categorization, and location categorization), which might have already consumed much of the resources available for mental control (Hübner, Futterer, \& Steinhauser, 2001). Thus, from these results it is hard to tell whether location-specific PC will occur in a pure Simon task or not. Therefore, we conducted an experiment to investigate this issue. The stimuli appeared not only left or right of fixation but also in different rows above or below 
fixation. While "left" and "right" were irrelevant, as usual, "above" and "below" were linked to a specific PC, respectively. Thus, a specific PC was not linked to an individual location but to a specific row on the screen. If individuals are able to use vertically separated regions in the visual field as cue for a specific control demand, and to adjust control parameters accordingly, then location-specific PC effects should occur. More specifically, in this case we should observe a significant interaction between PC and congruency (Simon effect).

\section{Methods}

\section{Participants}

Twenty students (mean age 21.7 years; 5 males) from the Universität Konstanz, Germany, participated in the experiment. All had normal or corrected-to-normal vision and were paid $8 €$ for their participation.

\section{Stimuli, apparatus, and procedure}

Stimuli were red or blue squares of size $7 \times 7 \mathrm{~mm}$. They were presented against a black background on an 18 " color-monitor with a resolution of $1280 \times 1024$ pixels and a refresh rate of $60 \mathrm{~Hz}$. Stimulus locations were defined by the four corners of a centered imaginary box of size $105 \times 105 \mathrm{~mm}$ on the screen. A centered white horizontal line of size $105 \times 1 \mathrm{~mm}$ separated the upper part of the box from the lower part. The line had a gap of $15 \mathrm{~mm}$ in the middle for positioning the fixation cross. Participants were seated in front of the screen at a viewing distance of approximately $60 \mathrm{~cm}$. Stimulus presentation as well as response registration was controlled by the same personal computer. Each trial started with the presentation of a fixation cross at the center of the screen for $400 \mathrm{~ms}$, followed by a blank screen for $400 \mathrm{~ms}$. Then, a single target stimulus randomly appeared for $165 \mathrm{~ms}$ either left and above fixation, left and below fixation, right and above fixation, or right and below fixation. The subsequent blank screen was shown until the response (Fig. 1). After displaying a blank screen for $1000 \mathrm{~ms}$, the next trial started.

The task was to indicate the color of the stimulus by pressing the corresponding mouse button (left button for blue; right button for red) with the index or middle finger of the right hand. The participants were instructed to respond as fast as possible without making many errors. On every incorrect trial, an auditory feedback was provided. At the end of each block, the mean error rate and response time in that block were displayed. If the mean error rate exceeded $10 \%$, the participants were asked to be more accurate.

In addition to 30 practice trials, there were 1,024 experimental trials. For half of the participants in each experimental block, $75 \%$ of stimuli were congruent and $25 \%$ incongruent (high PC) if they occurred in one of the top positions, whereas $25 \%$ of the stimuli were congruent and $75 \%$ incongruent (low PC) if they showed up at the bottom positions. This relation was reversed for the other half of participants. The overall proportion of congruent and incongruent trials within each block was $50 \%$, respectively. Participants were not informed about the relation between PC and stimulus location.

\section{Results}

\section{Response times}

Responses faster than $100 \mathrm{~ms}$ or slower than $2000 \mathrm{~ms}$ were excluded from analysis ( $<0.4 \%$ of all data). For examining the temporal development of the effects, we arranged the experimental trials into 8 subsequent blocks. This number guaranteed a minimum of 16 trials per condition. The latencies of correct responses were subjected to an ANOVA for repeated measurements on the factors congruency (congruent, or incongruent), $P C$ (proportion congruent: high, or low), and block (1 to 8).

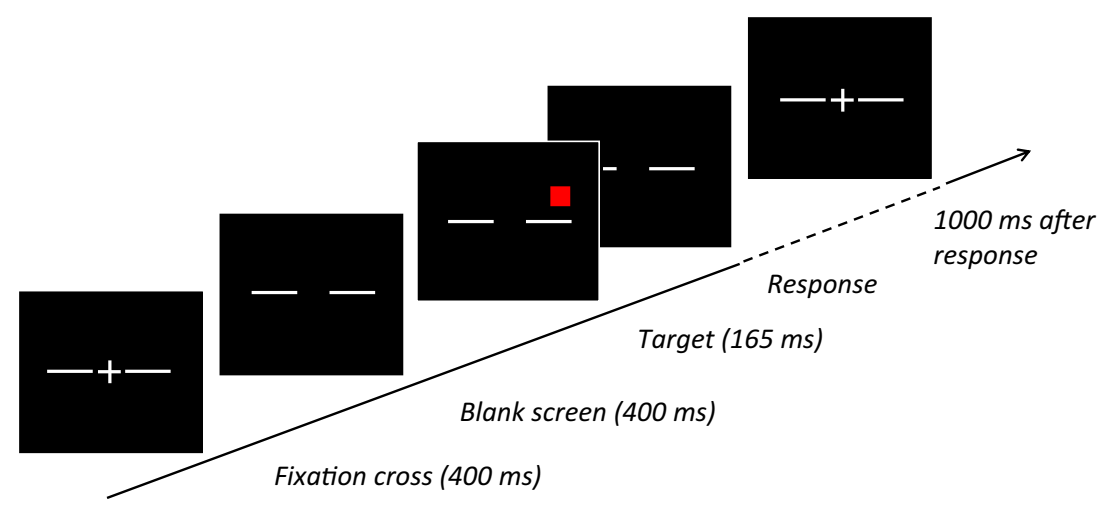

Fig. 1 Procedure of the experiment 
The analysis revealed a main effect of congruency, $F(1,19)$ $=81.0, p<0.001, \eta_{\mathrm{p}}^{2}=0.810$. Moreover, and most importantly, there was a significant interaction between congruency and $P C, F(1,19)=7.05, p<0.05, \eta_{\mathrm{p}}{ }^{2}=0.271$. The Simon effect was larger for the high-PC $(\Delta=29 \mathrm{~ms}, \mathrm{SD}=14.5)$ locations than for the low-PC $(\Delta=21 \mathrm{~ms}, \mathrm{SD}=13.1)$ ones (Fig. 2). Further analysis revealed that the Simon effect was also significant for the low-PC condition $t(19)=-7.11, p<$ 0.001 . The factor block also was significant $F(7,133)=2.36$, $p<0.05, \eta_{\mathrm{p}}{ }^{2}=0.111$. However, this factor was qualified by a reliable interaction with congruency $F(7,133)=2.50, p<$ $0.05, \eta_{\mathrm{p}}{ }^{2}=0.116$. As shown in Fig. 3 , the Simon effect decreased after the first two blocks. The interaction between all factors was far from significance, $F(7,133)=0.564, p=$ $0.784, \eta_{\mathrm{p}}^{2}=0.029$.

\section{Error rates}

Mean error rate was $6.58 \%$. The mean error rates were subjected to an ANOVA of the same type as for the latencies. It revealed a significant main effect of congruency, $F(1,19)=$ $84.2, p<0.001, \eta_{\mathrm{p}}{ }^{2}=0.816$, and also a significant interaction between congruency and $P C, F(1,19)=15.5, p<0.001, \eta_{\mathrm{p}}{ }^{2}=$ 0.450 . The Simon effect was larger for high-PC $(\Delta=7.27 \%$, $\mathrm{SD}=3.19)$ locations than for low-PC $(\Delta=3.46 \%, \mathrm{SD}=3.58)$

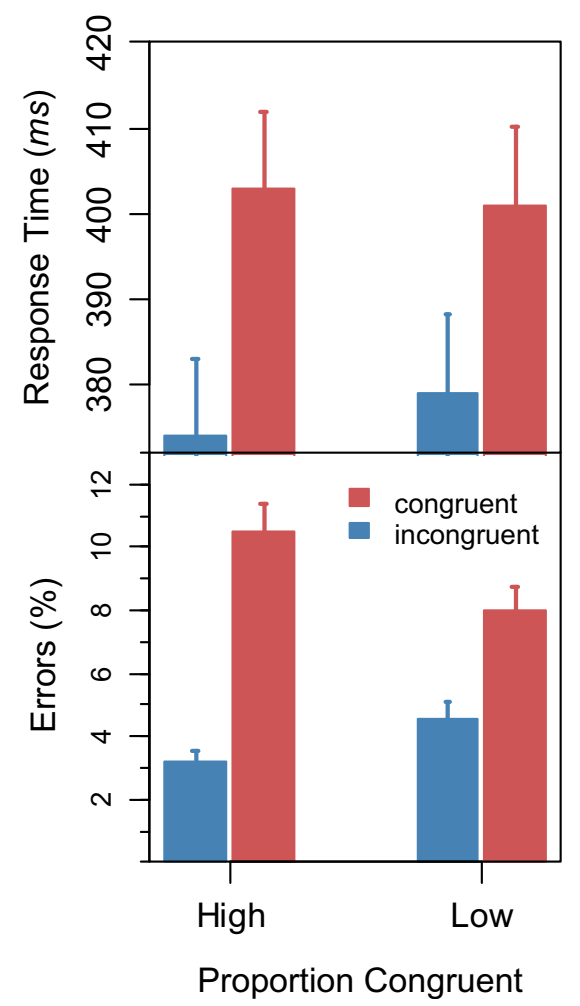

Fig. 2 Simon effect in the response times and error rates for the two proportion-congruent conditions. The error bars indicate the standard error

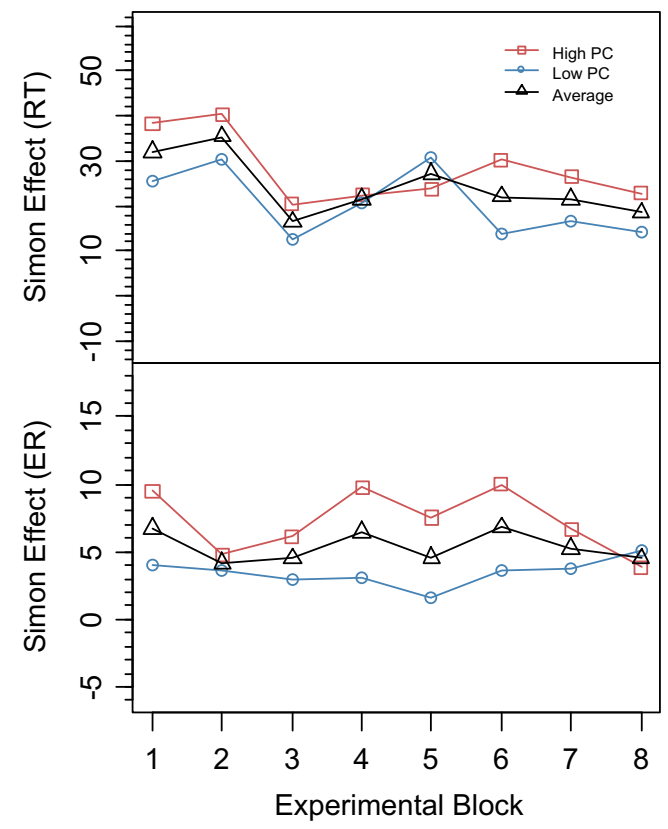

Fig. 3 Temporal development of the Simon effect in the two proportioncongruent conditions. The upper panel depicts the development in the response times (RT), whereas the lower panel shows the development in the error rates (ER)

ones (Fig. 2). Further analysis revealed that the Simon effect was also significant for the low-PC condition $t(19)=-4.33, p$ $<0.001$. The factor block produced no significant main effect or interactions. The interaction between all factors was far from significance, $F(7,133)=1.62, p=0.136, \eta_{\mathrm{p}}{ }^{2}=0.078$.

\section{Discussion}

We tested whether location-specific proportion-congruent (PC) effects also occur in the Simon task. Such PC effects are interesting, because they demonstrate that stimulus location can serve as context for adjusting attentional control parameters according to the demands related to the specific locations. As far as we know, these effects have been demonstrated up to now only for the Eriksen flanker task (Wendt et al., 2008) and the Stroop task (Crump et al., 2006). From these results, however, it cannot be inferred that locationspecific control also is possible for the Simon task, because this task differs from the other two in crucial aspects. For instance, in the Simon task stimulus location is already used for inducing the basic congruency effect. Thus, it was open whether location additionally can be utilized as cue for attentional control.

In the Simon task, irrelevant-response activation is usually induced by positions (left versus right) separated along the horizontal dimension. Therefore, in our experiment we linked PC to the vertical dimension. If the 
stimulus appeared above or below fixation, then PC was high or low, respectively, or vice versa. Because the stimulus at the same time also appeared left or right of fixation, each PC condition was not linked to a unique single location but to a corresponding upper or lower region (row). Our results show that there were location-specific $\mathrm{PC}$ effects in the latencies as well as in the error rates. The Simon effect was larger or smaller for stimuli appearing in the row associated with high or low PC, respectively. Although the effect was relatively small in the response times $(8 \mathrm{~ms})$, it was also present in the error rates (3.81\%). With the flanker task, Wendt, et al. (2008) found a larger effect $(20 \mathrm{~ms})$ in the response times, but no effect in the error rates. This is similar to Crump, et al. (2006), who used the Stroop task and observed only an effect $(16 \mathrm{~ms})$ in the latencies. Thus, it seems that the location-specific PC effect is of similar size in the three conflict paradigms.

As far as we know, our results demonstrate for the first time that location-specific PC effects also can occur in the Simon task. As for the other tasks, no specific learning condition or instruction is needed. This indicates that location also can be used in the Simon task as cue to adjust attentional control according to location-specific demands. However, there is an alternative interpretation of our results. As one reviewer pointed out, in our task, location was confounded with response. For instance, if the high-PC condition is linked to the top row, then a stimulus presented in the top-left location requires a left response in $75 \%$ of the cases, whereas such a response is required only in $25 \%$ of the cases if the stimulus appears in the bottom-left location. Such unequal joint frequencies allow the interpretation that learned location-response associations were responsible for our PC effects.

Although this interpretation cannot definitively be ruled out, we think that it is rather unlikely that the same response can differentially be associated to locations that are close to each other and that differ only in a dimension that is not part of the response code. That such locationresponse relations are not learned is indicated, for instance, by the results of Proctor et al. (2003). The interference produced in their study by mixing a locationirrelevant (Simon) and a location-relevant task was independent of whether or not the corresponding stimuli appeared in task-specific rows. Whereas it is conceivable that task switching prevents location-specific attentional control, e.g., by exhausting the control resources, it is hard to see why location-response associations should not be learned under such conditions. In this is case, however, the separation of stimulus locations should matter. Furthermore, if location-response associations had been learned in our study, then we also should have observed a gradual build-up of the location-specific PC effects over the course of the experiment, which, however, was neither the case in the latencies nor in the error rates. Thus, associative location-response learning is not supported by the current data.

However, as shown by Schmidt, De Houwer, and Besner (2010), contingencies also can be learned very rapidly. These researchers proposed that contingency learning proceeds by storing recent instances of stimuli and their response in episodic memory. On each trial, during stimulus processing, a number of matching instances are then retrieved and response expectancy is determined. To account for the present results, one would have to assume that the specific stimulus location is also part of the stored instances. Interestingly, Schmidt et al. (2010) further found that memory load impairs contingency learning, which not only supports their instancebased account but also could explain the absence of location-specific PC effects in Proctor et al. (2003) if one assumes that task mixing produced high memory load.

The idea of instance-based contingency learning also can be used to formulate a more detailed control account of our location-specific PC effects. Given that a certain control effort is experienced on each trial, depending on the specific vertical position, it is conceivable that the effort together with the vertical position is stored as instance in episodic memory. The matching instances retrieved during stimulus processing are then used to determine how much attentional control is needed on a trial.

Thus, the open question is what instances are stored in episodic memory. Are recent instances of locationresponse pairs stored or instances encompassing location and control effort? We prefer the latter idea, because it can explain location-based PC effects in all conflict paradigms by a common mechanism.

Taken together, our results demonstrate that location/ context-specific PC effects can occur in all major conflict paradigms. It should be noted that the common ground merely concerns the possibility to link control demands to locations and not the attentional control mechanisms as such, because these mechanisms largely differ between the paradigms. Whereas spatial attention controls the selection of relevant stimulus information in the flanker task (Hübner, et al., 2010), performance in the Stroop task is controlled by modulating the degree to which word reading processes are integrated with those for color-naming (Crump et al., 2006). Finally, for the Simon task it is widely assumed that performance is controlled by the suppression of irrelevant response activation (Hübner \& Mishra, 2013). Irrespective of which mechanism is involved, our data suggest that the control effort on a given trial is determined, at least partly, by the current stimulus location. 


\section{References}

Abrahamse, E. L., Duthoo, W., Notebaert, W., \& Risko, E. F. (2013). Attention modulation by proportion congruency: the asymmetrical list shifting effect. Journal of Experimental Psychology: Learning, Memory, and Cognition, 39, 1552.

Botvinick, M. M., Braver, T. S., Barch, D. M., Carter, C. S., \& Cohen, J. D. (2001). Conflict monitoring and cognitive control. Psychological Review, 108, 624-652.

Crump, M., Gong, Z., \& Milliken, B. (2006). The context specific proportion congruent stroop effect: Location as a contextual cue. Psychonomic Bulletin \& Review, 13, 316-321.

Eriksen, C. W., \& Schultz, D. W. (1979). Information processing in visual search: A continuous flow conception and experimental results. Perception \& Psychophysics, 25, 249-263.

Gratton, G., Coles, M. G., \& Donchin, E. (1992). Optimizing the use of information: Strategic control of activation of responses. Journal of Experimental Psychology: General, 121, 480-506.

Hasegawa, K., \& Takahashi, S. y. (2013). Functional difference between sustained and transient modulations of cognitive control in the Simon Task: Evidence from false alarm responses on no-go trials. PLOS ONE, 8, e81804.

Hommel, B. (1994). Spontaneous decay of response-code activation. Psychological Research, 56, 261-268.

Hübner, R., Futterer, T., \& Steinhauser, M. (2001). On attentional control as source of residual shift costs: Evidence from two-component task shifts. Journal of Experimental Psychology: Learning, Memory, and Cognition, 27, 640-653.

Hübner, R., \& Mishra, S. (2013). Evidence for strategic suppression of irrelevant activation in the Simon task. Acta Psychologica, 144, 166-172.

Hübner, R., Steinhauser, M., \& Lehle, C. (2010). A dual-stage two-phase model of selective attention. Psychological Review, 117, 759-784.

Lehle, C., \& Hübner, R. (2008). On-the-fly adaptation of selectivity in the flanker task. Psychonomic Bulletin \& Review, 15, 814-818.

Logan, G. D., \& Zbrodoff, N. J. (1979). When it helps to be misled: Facilitative effects of increasing the frequency of conflicting stimuli in an Stroop-like task. Memory \& Cognition, 3, 166-174.

Lu, C. H., \& Proctor, R. W. (1995). The influence of irrelevant location information on performance: A review of the Simon and spatial Stroop effects. Psychonomic Bulletin \& Review, 2, 174-207.
Marble, J. G., \& Proctor, R. W. (2000). Mixing location-relevant and location-irrelevant choice-reaction tasks: Influences of location mapping on the Simon effect. Journal of Experimental Psychology: Human Perception and Performance, 26, 1515-1533.

Proctor, R. W., Vu, K.-P. L., \& Marble, J. G. (2003). Mixing location-relevant and irrelevant tasks: Spatial compatibility effects eliminated by stimuli that share the same spatial codes. Visual Cognition, 10, 15-50.

Schmidt, J. R., De Houwer, J., \& Besner, D. (2010). Contingency learning and unlearning in the blink of an eye: A resource dependent process. Consciousness and Cognition, 19, 235-250.

Simon, J. R. (1969). Reactions toward the source of stimulation. Journal of Experimental Psychology, 81, 174-176.

Steinhauser, M., \& Hübner, R. (2009). Distinguishing Response Conflict and Task Conflict in the Stroop Task: Evidence From Ex-Gaussian Distribution Analysis. Journal of Experimental Psychology: Human Perception and Performance, 35, 1398-1412.

Stroop, J. R. (1935). Studies of interference in serial verbal reactions. Journal of Experimental Psychology, 18, 643-662.

Stürmer, B., Leuthold, H., Soetens, E., Schröter, H., \& Sommer, W. (2002). Control over location-based response activation in the Simon task: behavioral and electrophysiological evidence. Journal of Experimental Psychology: Human Perception and Performance, 28, 1345-1363.

Töbel, L., Hübner, R., \& Stürmer, B. (2014). Suppression of irrelevant activation in the horizontal and vertical Simon task differs quantitatively not qualitatively. Acta Psychologica, 152, 47-55.

Toth, J. P., Levine, B., Stuss, D. T., Oh, A., Winocur, G., \& Meiran, N. (1995). Dissociation of processes underlying spatial SR compatibility: Evidence for the independent influence of what and where. Consciousness and Cognition, 4, 483-501.

Vietze, I., \& Wendt, M. (2009). Context specificity of conflict frequencydependent control. Quarterly Journal of Experimental Psychology, 62, 1391-1400.

Wendt, M., Kluwe, R. H., \& Vietze, I. (2008). Location-specific versus hemisphere-specific adaptation of processing selectivity. Psychonomic Bulletin \& Review, 15, 135-140. 